\title{
MULTIPLE PERIODIC SOLUTIONS TO A CLASS OF SECOND-ORDER NONLINEAR MIXED-TYPE FUNCTIONAL DIFFERENTIAL EQUATIONS
}

\author{
XIAO-BAO SHU AND YUAN-TONG XU
}

Received 22 October 2004 and in revised form 23 August 2005

By means of variational structure and $Z_{2}$ group index theory, we obtain multiple periodic solutions to a class of second-order mixed-type differential equations $x^{\prime \prime}(t-\tau)+$ $f(t, x(t), x(t-\tau), x(t-2 \tau))=0$ and $x^{\prime \prime}(t-\tau)+\lambda(t) f_{1}(t, x(t), x(t-\tau), x(t-2 \tau))=x(t-\tau)$.

\section{Introduction}

Recently, the existence and multiplicity of periodic solutions for second-order functional differential equations has received a great deal of attention (e.g., see [8, 9, 12]). In [9], Wang and Yan studied the second-order functional differential equation

$$
[x(t)+c x(t-\tau)]^{\prime \prime}+g(t, x(t-\sigma))=p(t),
$$

where $\tau, \sigma$, and $c$ are constants in R with $\tau \geq 0, \sigma \geq 0,|c|<1, g(t, x)$ is a $T(>0)$-periodic function in $t>0$, and for an arbitrary bounded domain $E \subset R, g(t, x)$ is a Lipschitz function in $[0, T] \times E, p \in C(R, R), p(t+T)=p(t)$, and $\int_{0}^{T} p(t) d t=0$. They obtained some sufficient conditions to guarantee the existence, at least a T-periodic solution, for this system.

But, for the existence of periodic solutions of functional differential equations, one commonly uses methods of fixed point theory, coincidence degree theory, Fourier analysis, and so forth. Critical point theory has rarely been used. In $[10,11]$, the authors obtained multiple periodic solutions for a class retarded differential equations by means of critical point theory and $Z_{p}$ group index theory. Nevertheless, we noted that these results were obtained by reducing retarded differential equations to related ordinary differential equations.

The purpose of our paper is to establish a kind of variational framework with delayed variables for a class of mixed-type differential equations. Unlike $[10,11]$, our approach enables us to obtain, by critical point theory and $Z_{2}$ group index theory, the existence of nontrivial periodic solutions to such equations without reducing it to the one of ordinary differential equations. Subsequently, we introduce $Z_{2}$ group index theory and knowledge about critical points. 
Definition 1.1. A critical point of $f$ is a point where $f^{\prime}(x)=0$. A critical value of $f$ is a number $c$ such that $f(x)=c$ for some critical points $x$. $K$ is critical set where $K=\{x \in$ $\left.E \mid f^{\prime}(x)=0\right\}, K_{c}=\left\{x \in E \mid f^{\prime}(x)=0, f(x)=c\right\} . f_{c}$ is a level set if $f_{c}=\{x \in E \mid f(x) \leq c\}$.

Definition 1.2. Let $E$ be real Banach space, and $f \in C^{1}(E, R)$, we say that $f$ satisfies the Palais-Smale condition if every sequence $\left\{x_{n}\right\} \subset E$ such that $\left\{f\left(x_{n}\right)\right\}$ is bounded and $f^{\prime}\left(x_{n}\right) \rightarrow \theta(n \rightarrow \infty)$ has a converging subsequence.

Definition 1.3. Let $E$ be real Banach space, and $\sum=\{A \mid A \subset E \backslash\{\theta\}$ is closed, symmetric set\}. Define $\gamma: \sum \rightarrow Z^{+} \bigcup\{+\infty\}$ as following:

$$
\gamma(A)= \begin{cases}\min & \left\{n \in Z: \text { there exists an odd continuous map } \varphi: A \longrightarrow R^{n} \backslash\{\theta\}\right\} \\ 0 & \text { if } A=\varnothing ; \\ +\infty & \text { if there is no odd continuous map } \varphi: A \longrightarrow R^{n} \backslash\{\theta\} \text { for any } n \in Z .\end{cases}
$$

Then we say $\gamma$ is the genus of $\sum$. Denote $i_{1}(f)=\lim _{a \rightarrow-0} \gamma\left(f_{a}\right)$ and $i_{2}(f)=\lim _{a \rightarrow-\infty} \gamma\left(f_{a}\right)$. Lemma 1.4 (Rabinowitz [7]). Let $f \in C^{1}\left(X, R^{1}\right)$ be an even functional which satisfies the Palais-Smale condition and $f(\theta)=0$. If

(i) there exists $\rho>0, \alpha>0$, and a finite dimensional subspace E of $X$, such that $\left.f\right|_{E^{\perp} \cap S_{\rho}} \geq$ $\alpha$.

(ii) for all finite dimensional subspaces $\tilde{E}$ of $X$, there is an $r=r(\widetilde{E})>0$, such that $f(x) \leq$ 0 for $x \in \tilde{E} \backslash B_{r}$.

Then, $f$ possesses an unbounded sequence of critical values.

Lemma 1.5 (Chang [1]). Let $f \in C^{1}\left(E, R^{1}\right)$ be an even functional which satisfies the PalaisSmale condition and $f(\theta)=0$. Then,

$\left(\mathrm{P}_{1}\right)$ If there exists an $m$-dimensional subspace $X$ of $E$ and $\rho>0$ such that

$$
\sup _{x \in X \cap S_{\rho}} f(x)<0
$$

then we have $i_{1}(f) \geq m$;

$\left(\mathrm{P}_{2}\right)$ If there exists an $j$-dimensional subspace $\tilde{X}$ of $E$ such that

$$
\inf _{x \in \widetilde{X}^{\perp}} f(x)>-\infty
$$

we have $i_{2}(f) \leq j$.

If $m \geq j$, and $\left(\mathrm{P}_{1}\right)$ and $\left(\mathrm{P}_{2}\right)$ hold, then $f$ at least has $2(m-j)$ distinct critical points.

Lemma 1.6. Let $E$ be Hilbert space, if the weak convergence sequence $\left\{x_{n}\right\} \subset E$ satisfies $\left\|x_{n}\right\| \rightarrow\left\|x_{0}\right\|(n \rightarrow \infty)$, then $\left\{x_{n}\right\}$ is convergent in $E$, that is, $x_{n} \rightarrow x_{0}$. 
Proof. By

$$
\begin{aligned}
\left\|x_{n}-x_{0}\right\|^{2} & =\left(x_{n}-x_{0}, x_{n}-x_{0}\right) \\
& =\left\|x_{n}\right\|^{2}-\left(x_{0}, x_{n}\right)-\left(x_{n}, x_{0}\right)+\left\|x_{0}\right\| \quad(n=1,2,3, \ldots)
\end{aligned}
$$

and continuity of inner product, it is easy to see that

$$
\lim _{n \rightarrow \infty}\left\|x_{n}-x_{0}\right\|^{2}=\left\|x_{0}\right\|^{2}-2\left(x_{0}, x_{0}\right)+\left\|x_{0}\right\|^{2}=0,
$$

that is, $x_{n} \rightarrow x_{0}(n \rightarrow \infty)$.

First, we use Lemma 1.4 to deal with multiple periodic solutions of the following second-order mixed-type delay equations

$$
x^{\prime \prime}(t-\tau)+f(t, x(t), x(t-\tau), x(t-2 \tau))=0 .
$$

Our basic assumptions are that

$\left(\mathrm{A}_{1}\right) f\left(t, u_{1}, u_{2}, u_{3}\right) \in C\left(R^{4}, R\right)$ and $\partial f\left(t, u_{1}, u_{2}, u_{3}\right) / \partial t \neq 0$, as well as there exists a continuous function $g(t, u, v) \in C\left(R^{3}, R\right)$ that satisfies $\partial g / \partial u$, and $\partial g / \partial v$ are well defined such that

$$
f\left(t, u_{1}, u_{2}, u_{3}\right)=g\left(t, u_{1}, u_{2}\right)+\int_{0}^{u_{3}} g_{u_{2}}^{\prime}\left(t, u_{2}, \omega\right) d \omega-u_{2}
$$

$\left(\mathrm{A}_{2}\right) f\left(t+\tau, u_{1}, u_{2}, u_{3}\right)=f\left(t, u_{1}, u_{2}, u_{3}\right)$,

$$
f\left(t,-u_{1},-u_{2},-u_{3}\right)=-f\left(t, u_{1}, u_{2}, u_{3}\right) .
$$

\section{Variational structure}

Let

$$
\begin{aligned}
H_{2 \gamma \tau}^{1}= & \left\{x(t) \in L^{2}[0,2 \gamma \tau] \mid x^{\prime}(t) \in L^{2}[0,2 \gamma \tau] x(t) \text { is } 2 \gamma \tau \text {-periodic functionin } t,\right. \\
& \text { where } \gamma \text { is a given positive integer }\} .
\end{aligned}
$$

It is obvious that $H_{2 \gamma \tau}^{1}$ is a Sobolev space by defining the inner product $(\cdot, \cdot)$ and the norm $\|\cdot\|$,

$$
\begin{gathered}
\langle x, y\rangle_{H_{2 \gamma \tau}^{1}}=\int_{0}^{2 \gamma \tau}\left[x(t) y(t)+x^{\prime}(t) y^{\prime}(t)\right] d t \\
\|x\|_{H_{2 \gamma \tau}^{1}}=\left|\int_{0}^{2 \gamma \tau}\left[|x(t)|^{2}+\left|x^{\prime}(t)\right|^{2}\right] d t\right|^{1 / 2}, \quad \forall x, y \in H_{2 \gamma \tau}^{1},
\end{gathered}
$$

as well as $x(t)$ can be expressed as

$$
x(t)=a_{0}+\sum_{k=1}^{\infty}\left(a_{k} \cos \frac{k \pi}{\gamma \tau} t+b_{k} \sin \frac{k \pi}{\gamma \tau} t\right) .
$$


Let us consider the function defined on $H_{2 \gamma \tau}^{1}$,

$$
I(x)=\int_{0}^{2 \gamma \tau}\left[\frac{1}{2}\left(\left|x^{\prime}(t)\right|^{2}+|x(t)|^{2}\right)-\int_{0}^{x(t-\tau)} g(t, x(t), \omega) d \omega\right] d t
$$

Then, for all $x, y \in H_{2 \gamma \tau}^{1}$ and $\varepsilon>0$, we know that

$$
\begin{aligned}
I(x+\varepsilon y)=I(x)+\varepsilon\left(\int_{0}^{2 \gamma \tau}\left[x(t) y(t)+x^{\prime}(t) y^{\prime}(t)\right] d t\right. \\
-\int_{0}^{2 \gamma \tau}\left[\left(\int_{0}^{x(t-\tau)} g_{u_{1}}^{\prime}(t, x(t)+\varepsilon \theta(t) y(t), \omega) d \omega\right) y(t)\right. \\
\quad+g(t, x(t), x(t-\tau)+\varepsilon y(t-\tau)) y(t-\tau)] d t) \\
+\frac{\varepsilon^{2}}{2} \int_{0}^{2 \gamma \tau}\left[y^{2}(t)+\left|y^{\prime}(t)\right|^{2}\right] d t,
\end{aligned}
$$

where $0 \leq \theta(t) \leq 1$. It is easy to see that

$$
\begin{aligned}
\left\langle I^{\prime}(x), y\right\rangle= & \int_{0}^{2 \gamma \tau}\left[x^{\prime}(t) y^{\prime}(t)+x(t) y(t)-\int_{0}^{x(t-\tau)} g_{u_{1}}^{\prime}(t, x(t), \omega) d \omega\right] y(t) d t \\
& -\int_{0}^{2 \gamma \tau} g(t, x(t), x(t-\tau)) y(t-\tau) d t .
\end{aligned}
$$

By the periodicity $g(t, u, v), x(t), x(t-\tau)$, and $y(t)$, we get that

$$
\begin{aligned}
\int_{0}^{2 \gamma \tau} g(t, x(t), x(t-\tau)) y(t-\tau) d t & =\int_{-\tau}^{(2 \gamma-1) \tau} g(t+\tau, x(t+\tau), x(t)) y(t) d t \\
& =\int_{0}^{2 \gamma \tau} g(t, x(t+\tau), x(t)) y(t) d t .
\end{aligned}
$$

Hence,

$$
\begin{aligned}
\left\langle I^{\prime}(x), y\right\rangle=\int_{0}^{2 \gamma \tau}[ & -x^{\prime \prime}(t)+x(t)-\int_{0}^{x(t-\tau)} g_{u_{1}}^{\prime}(t, x(t), \omega) d \omega \\
& -g(t, x(t+\tau), x(t))] y(t) d t .
\end{aligned}
$$

Therefore, the Euler equation corresponding to the function $I(x)$ is as follows:

$$
x^{\prime \prime}(t)-x(t)+\int_{0}^{x(t-\tau)} g_{u_{1}}^{\prime}(t, x(t), \omega) d \omega+g(t, x(t+\tau), x(t))=0 .
$$

It is easy to see that (2.9) is equivalent to (1.7), so the system (1.7) is the Euler equation corresponding to the function $I(x)$. Then, we may get $2 \gamma \tau$-periodic solutions of the system (1.7), by seeking critical points of the function $I(x)$. 


\section{Main results}

THEOREM 3.1. Under the assumptions $\left(\mathrm{A}_{1}\right) \sim\left(\mathrm{A}_{2}\right)$ and the function $g\left(t, u_{1}, u_{2}\right)$ satisfying the following conditions:

$\left(\mathrm{C}_{1}\right)$ there exists a constant $T>0$ such that

$$
\lim _{|u| \rightarrow 0} \frac{\int_{0}^{u_{2}} g\left(t, u_{1}, \omega\right) d \omega}{|u|^{2}} \leq T,
$$

where $|u|=\sqrt{u_{1}^{2}+u_{2}^{2}}$.

$\left(\mathrm{C}_{2}\right)$ there exist constants $\beta>2$ and $\alpha>0$ such that

$$
\begin{aligned}
0 & <G\left(t, u_{1}, u_{2}\right)=\int_{0}^{u_{2}} g\left(t, u_{1}, \omega\right) d \omega \\
& \leq \frac{1}{\beta}\left[\left(\int_{0}^{u_{2}} g_{u_{1}}^{\prime}\left(t, u_{1}, \omega\right) d \omega\right) u_{1}+g\left(t, u_{1}, u_{2}\right) u_{2}\right], \quad \forall|u|=\sqrt{u_{1}^{2}+u_{2}^{2}} \geq \alpha,
\end{aligned}
$$

then the problem (1.7) has an infinite number of nontrivial $2 \gamma \tau$-periodic solutions.

It is not difficult to see that if $x(t)$ is a solution of the system (1.7), then $-x(t)$ is also a solution of the system (1.7) by the assumption $\left(\mathrm{A}_{2}\right)$. That is, the solution of the system (1.7) is a set that is symmetric with respect to the origin in $H_{2 \gamma \tau}^{1}$. On the other hand, if we let $\eta(t, x)=G(t, x(t), x(t-\tau))=\int_{0}^{x(t-\tau)} g(t, x(t), \omega) d \omega$, it is easy to see that $\eta(t, x)$ is an even function in $x$, so $I(x)$ is an even function in $x$ and we may show that Theorem 3.1 holds by Lemma 1.4 .

In order to exploit Lemma 1.4 to find the critical points of function $I(x)$ in $(2.4)$, one needs to verify all the assumptions. First of all, we point out that the functional $I(\cdot)$, defined on $H_{2 \gamma \tau}^{1}$, satisfies the Palais-Smale condition, that is, we have the following lemma.

LEMMA 3.2. Under the assumptions $\left(\mathrm{A}_{1}\right) \sim\left(\mathrm{A}_{2}\right)$ and the conditions $\left(\mathrm{C}_{1}\right) \sim\left(\mathrm{C}_{2}\right), I(u)$ satisfies the P. S. condition.

Proof. Let $\left\{u_{n}\right\} \subset H_{2 \gamma \tau}^{1}$ and the constants $c_{1}, c_{2}$ satisfy

$$
\begin{gathered}
c_{1} \leq I\left(u_{n}\right) \leq c_{2}, \\
I^{\prime}\left(u_{n}\right) \longrightarrow 0, \quad(n \longrightarrow \infty) .
\end{gathered}
$$

The above inequality (3.3) is equivalent to

$$
c_{1}<\int_{0}^{2 \gamma \tau}\left[\frac{1}{2}\left(\left|u_{n}^{\prime}(t)\right|^{2}+\left|u_{n}(t)\right|^{2}\right)-\int_{0}^{u_{n}(t-\tau)} g(t, x(t), \omega) d \omega\right] d t<c_{2} .
$$

Replacing $x$ and $y$ by $u_{n}$ in (2.6), we have

$$
\begin{aligned}
\left\|u_{n}\right\|_{H_{2 \gamma \tau}^{1}}^{2}= & \int_{0}^{2 \gamma \tau}\left[\left(\int_{0}^{u_{n}(t-\tau)} g_{\xi_{1}}^{\prime}\left(t, u_{n}(t), \omega\right) d \omega\right) u_{n}(t)\right. \\
& \left.+g\left(t, u_{n}(t), u_{n}(t-\tau)\right) u_{n}(t-\tau)\right] d t \\
& +\left\langle I^{\prime}\left(u_{n}\right), u_{n}\right\rangle .
\end{aligned}
$$


By (3.6), we have

$$
\begin{aligned}
& \int_{0}^{2 \gamma \tau}\left[\left(\int_{0}^{u_{n}(t-\tau)} g_{\xi_{1}}^{\prime}\left(t, u_{n}(t), \omega\right) d \omega\right) u_{n}(t)+g\left(t, u_{n}(t), u_{n}(t-\tau)\right) u_{n}(t-\tau)\right] d t \\
& \quad=\left\|u_{n}\right\|_{H_{2 \gamma \tau}^{1}}^{2}-\left\langle I_{n}^{\prime}\left(u_{n}\right), u_{n}\right\rangle .
\end{aligned}
$$

Next, we show that a sequence $\left\{u_{n}\right\}$ satisfying condition (3.3) and (3.4) is bounded. Denote $B_{1}=\left\{t \in[0,2 \gamma \tau]|| u_{n}(t) \mid=\sqrt{u_{n}^{2}(t)+u_{n}^{2}(t-\tau)} \geq \alpha\right\}, B_{2}=[0,2 \gamma \tau] \backslash B_{1}$. By the condition $\left(\mathrm{C}_{2}\right)$ and (3.6), we have

$$
\begin{aligned}
& I\left(u_{n}\right)= \frac{1}{2}\left\|u_{n}\right\|^{2}-\int_{0}^{2 \gamma \tau}\left(\int_{0}^{u_{n}(t-\tau)} g\left(t, u_{n}(t), \omega\right) d \omega\right) d t \\
&= \frac{1}{2}\left\|u_{n}\right\|^{2}-\int_{B_{1}} G\left(t, u_{n}(t), u_{n}(t-\tau)\right) d t-\int_{B_{2}} G\left(t, u_{n}(t), u_{n}(t-\tau)\right) d t \\
& \geq \frac{1}{2}\left\|u_{n}\right\|^{2}-\int_{B_{1}} \frac{1}{\beta}\left[\left(\int_{0}^{u_{n}(t-\tau)} g_{\xi_{1}}^{\prime}\left(t, u_{n}(t), \omega\right) d \omega\right) u_{n}(t)\right. \\
&\left.+g\left(t, u_{n}(t), u_{n}(t-\tau)\right) u_{n}(t-\tau)\right] d t-c_{3} \\
& \geq \frac{1}{2}\left\|u_{n}\right\|^{2}-\frac{1}{\beta} \int_{0}^{2 \gamma \tau}\left[\left(\int_{0}^{u_{n}(t-\tau)} g_{\xi_{1}}^{\prime}\left(t, u_{n}(t), \omega\right) d \omega\right) u_{n}(t)\right. \\
&\left.\quad+g\left(t, u_{n}(t), u_{n}(t-\tau)\right) u_{n}(t-\tau)\right] d t-c_{4} \\
&=\frac{1}{2}\left\|u_{n}\right\|^{2}-\frac{1}{\beta}\left[\left\|u_{n}\right\|^{2}-\left\langle I^{\prime}\left(u_{n}\right), u_{n}\right\rangle\right]-c_{4} \\
& \geq\left(\frac{1}{2}-\frac{1}{\beta}\right)\left\|u_{n}\right\|^{2}-\frac{1}{\beta}\left\|I^{\prime}\left(u_{n}\right)\right\|\left\|u_{n}\right\|-c_{4} .
\end{aligned}
$$

Remarks 3.3. In here and the following, $c_{i}>0$.

Then, by (3.3) and (3.4), it is easy to see

$$
\begin{aligned}
\left(\frac{1}{2}-\frac{1}{\beta}\right)\left\|u_{n}\right\|^{2} & \leq \frac{1}{\beta}\left\|I^{\prime}\left(u_{n}\right)\right\|\left\|u_{n}\right\|+c_{4} \\
& \leq c_{5}\left\|u_{n}\right\|+c_{6} .
\end{aligned}
$$

Since $\beta>2$, we know that $\left\{\left\|u_{n}\right\|\right\}$ is bounded.

Since $H_{2 \gamma \tau}^{1}$ is a reflexive Banach space and the sequence $\left\{u_{n}\right\}$ is bounded, so $\left\{u_{n}\right\}$ has a weakly convergent subsequence. We still denote it by $\left\{u_{n}\right\}$ and suppose that $u_{n} \rightarrow u_{0}$ in $H_{2 \gamma \tau}^{1}$ as $n \rightarrow \infty$.

So by (3.7) and the boundedness of $\left\|u_{n}\right\|$, we get that

$$
\begin{aligned}
& \left\|u_{n}\right\|_{H_{2 \gamma \tau}^{1}}^{2}-\int_{0}^{2 \gamma \tau}\left(\int_{0}^{u_{n}(t-\tau)} g_{\xi_{1}}^{\prime}\left(t, u_{n}(t), \omega\right) d \omega\right) u_{n}(t) d t \\
& \quad-\int_{0}^{2 \gamma \tau} g\left(t, u_{n}(t), u_{n}(t-\tau)\right) u_{n}(t-\tau) d t \longrightarrow 0, \quad(n \longrightarrow \infty) .
\end{aligned}
$$


On the other hand, the weak convergence of $\left\{u_{n}\right\}$ of $H_{2 \gamma \tau}^{1}$ implies the uniform convergence of $\left\{u_{n}\right\}$ in $C([0,1], R)$ (see $\left.[6]\right)$. Hence,

$$
\begin{aligned}
\left\|u_{n}\right\|_{H_{2 \gamma \tau}^{1}}^{2} \longrightarrow & \int_{0}^{2 \gamma \tau}\left(\int_{0}^{u_{0}(t-\tau)} g_{\xi_{1}}^{\prime}\left(t, u_{0}(t), \omega\right) d \omega\right) u_{0}(t) d t \\
& +\int_{0}^{2 \gamma \tau} g\left(t, u_{0}(t), u_{0}(t-\tau)\right) u_{0}(t-\tau) d t, \quad(n \longrightarrow \infty) .
\end{aligned}
$$

This means that $\left\|u_{n}\right\|$ is convergent in $H_{2 \gamma \tau}^{1}$, then, by Lemma 1.6, we get that the function $I$ satisfies the P. S. condition.

Lemma 3.4. Under the assumptions $\left(A_{1}\right) \sim\left(A_{2}\right)$ and the conditions $\left(C_{1}\right) \sim\left(C_{2}\right)$, then there exist $\rho, \alpha>0$ and finite dimensional subspace $E$ of $H_{2 \gamma \tau}^{1}$, such that

$$
I(x)_{E^{\perp} \cap S_{\rho}} \geq \alpha .
$$

Proof. Let $v_{j}(t)=(\gamma \tau / k \pi) \sin (k \pi / \gamma \tau) t, j=1,2, \ldots$, then

$$
\begin{aligned}
& \int_{0}^{2 \gamma \tau}\left|v_{j}(t)\right|^{2} d t=\frac{\gamma^{2} \tau^{2}}{k^{2} \pi^{2}} \gamma \tau, \\
& \int_{0}^{2 \gamma \tau}\left|v_{j}^{\prime}(t)\right|^{2} d t=\gamma \tau .
\end{aligned}
$$

Define an $n$-dimensional linear space as follows:

$$
E=\operatorname{span}\left\{v_{1}, v_{2}, \ldots, v_{n}\right\}, \quad X=E^{\perp} .
$$

For all $x(t) \in S_{\rho} \cap X$, we get that

$$
\begin{aligned}
|x(t)| & \leq\left|\int_{0}^{2 \gamma \tau} x^{\prime}(t) d t\right| \leq \int_{0}^{2 \gamma \tau}\left|x^{\prime}(t)\right| d t \\
& \leq \sqrt{2 \gamma \tau}\left(\int_{0}^{2 \gamma \tau}\left|x^{\prime}(t)\right|^{2} d t\right)^{1 / 2} \leq \sqrt{2 \gamma \tau}\|x\|_{H_{2 \gamma \tau}^{1}}
\end{aligned}
$$

By the periodicity of $x(t)$, it is easy to see that $|x(t-\tau)| \leq \sqrt{2 \gamma \tau}\|x\|_{H_{2 \tau}^{1}}$. On the other hand, by the condition $\left(\mathrm{C}_{2}\right)$, for all $\varepsilon_{0}>0$, there exists $\delta>0$, such that when $u=(x(t), x(t-\tau))$ satisfies

$$
x^{2}(t)+x^{2}(t-\tau) \leq 2\left(\sqrt{2 \gamma \tau}\|x\|_{H_{2 \gamma \tau}^{1}}\right)^{2}=4 \gamma \tau\|x\|_{H_{2 \tau}^{1}}^{2} \leq \delta^{2},
$$

we have

$$
|G(t, x(t), x(t-\tau))|=\int_{0}^{x(t-\tau)} g(t, x(t), \omega) d \omega \leq\left(T+\varepsilon_{0}\right)\left[x^{2}(t)+x^{2}(t-\tau)\right] .
$$


At the same time, it is not difficult to see that

$$
\int_{0}^{2 \gamma \tau}|x(t)|^{2} d t \leq \frac{\gamma^{2} \tau^{2}}{k^{2} \pi^{2}} \int_{0}^{2 \gamma \tau}\left|x^{\prime}(t)\right|^{2} d t
$$

holds when $x(t) \in S_{\rho} \cap X$. So one gets

$$
\int_{0}^{2 \gamma \tau}|x(t)|^{2} d t \leq \frac{\gamma^{2} \tau^{2}}{k^{2} \pi^{2}+\gamma^{2} \tau^{2}} \rho^{2}
$$

By the above equality and (3.17) as well as the periodicity of $x(t-\tau)$, we have

$$
\begin{aligned}
I(x) & =\int_{0}^{2 \gamma \tau}\left[\frac{1}{2}\left(\left|x^{\prime}(t)\right|^{2}+|x(t)|^{2}\right)-\int_{0}^{x(t-\tau)} g(t, x(t), x(t-\tau))\right] d t \\
& \geq \frac{1}{2}\|x\|_{H_{2 \gamma \tau}^{1}}^{2}-2\left(T+\varepsilon_{0}\right) \int_{0}^{2 \gamma \tau}|x(t)|^{2} d t \\
& \geq \frac{1}{2} \rho^{2}-\frac{2\left(T+\varepsilon_{0}\right) \gamma^{2} \tau^{2}}{k^{2} \pi^{2}+\gamma^{2} \tau^{2}} \rho^{2}=\frac{1}{2}\left(1-\frac{4\left(T+\varepsilon_{0}\right) \gamma^{2} \tau^{2}}{k^{2} \pi^{2}+\gamma^{2} \tau^{2}}\right) \rho^{2}>0 .
\end{aligned}
$$

Remark 3.5. We may choose $T>0$ and $\varepsilon_{0}>0$ such that the above equality holds.

That is, Lemma 3.4 holds.

Lemma 3.6. Under the assumptions $\left(\mathrm{A}_{1}\right) \sim\left(\mathrm{A}_{2}\right)$ and the conditions $\left(\mathrm{C}_{1}\right) \sim\left(\mathrm{C}_{2}\right)$, for all finite dimensional subspace $\widetilde{E}$ of $H_{2 \gamma \tau}^{1}$, there is an $R=R(\widetilde{E})>0$, such that

$$
I(x) \leq 0, \quad \forall x \in \widetilde{E} \backslash B_{R} .
$$

Proof. For an arbitrary finite dimensional subspace $E_{1} \subset H_{2 \gamma \tau}^{1}$, by $\left(\mathrm{C}_{2}\right)$, we know that there exist constant $\alpha_{1}>0$ and $\alpha_{2}>0$ such that

$$
\int_{0}^{x(t-\tau)} g(t, x(t), \omega) d \omega \geq \alpha_{1}\left|\sqrt{u_{1}^{2}+u_{2}^{2}}\right|^{\beta}-\alpha_{2}
$$

So, for any given $\varphi \in E_{1},\|\varphi\|_{H_{2 \gamma \tau}^{1}}=1$ and $\mu>0$, we have

$$
\begin{aligned}
I(\mu \varphi)= & \frac{1}{2} \mu^{2}\|\varphi\|_{H_{2 \gamma \tau}^{1}}^{2}-\int_{0}^{2 \gamma \tau}\left(\int_{0}^{\mu \varphi(t-\tau)} g(t, \mu \varphi(t), \omega) d \omega\right) d t \\
& \leq \frac{1}{2} \mu^{2}\|\varphi\|_{H_{2 \gamma \tau}^{1}}^{2}-\alpha_{1} \mu^{\beta} \int_{0}^{2 \gamma \tau}\left|\sqrt{\varphi^{2}(t)+\varphi^{2}(t-\tau)}\right|^{\beta} d t+2 \tau \alpha_{2} \longrightarrow-\infty, \quad \mu \longrightarrow+\infty
\end{aligned}
$$

Then there exists $\mu_{0}>0$. 
Remark 3.7. In fact, $\mu_{0}$ is a minimum value of $\mu$ that the above inequality holds on the unit ball of the finite dimensional subspace $E_{1}$.

For any given $\varphi \in E_{1},\|\varphi\|_{H_{2 \gamma \tau}^{1}}=1$, when $\mu \geq \mu_{0}$, such that $I(\mu \varphi)<0$. So choosing $R=$ $\mu_{0}$, we get that

$$
I(x) \leq 0, \quad \forall x \in \widetilde{E_{1}} \backslash B_{R}
$$

Since $E_{1}$ was arbitrary, we know Lemma 3.6 holds.

By $\left(\mathrm{A}_{2}\right)$, we get that $I(\theta)=0$. So, by Lemmas 3.2, 3.4, and 3.6, we know that $I$ has infinite nontrivial critical points, that is, the problem (1.7) has infinite nontrivial $2 \gamma \tau$ periodic solutions.

We next consider the nonlinear mixed-type delay equations

$$
x^{\prime \prime}(t-\tau)+\lambda(t) f_{1}(t, x(t), x(t-\tau), x(t-2 \tau))=x(t-\tau), \quad \lambda(t)>0
$$

Our basic assumptions is that

$\left(\mathrm{A}_{1}^{\prime}\right) f_{1}\left(t, u_{1}, u_{2}, u_{3}\right) \in C\left(R^{4}, R\right)$, and $\partial f_{1}\left(t, u_{1}, u_{2}, u_{3}\right) / \partial t \neq 0$, and there exists a continuous function $g_{1}(t, u, v) \in C\left(R^{2}, R\right)$ such that

$$
f_{1}\left(t, u_{1}, u_{2}, u_{3}\right)=g_{1}\left(t, u_{1}, u_{2}\right)+\int_{0}^{u_{3}} g_{1 u_{2}}^{\prime}\left(t, u_{2}, \omega\right) d \omega
$$

$\left(\mathrm{A}_{2}^{\prime}\right) f\left(t+\tau, u_{1}, u_{2}, u_{3}\right)=f\left(t, u_{1}, u_{2}, u_{3}\right)$, and $\lambda(t) \in C(R, R)$ satisfies $\lambda(t+\tau)=\lambda(t)$ as well as

$$
f_{1}\left(t,-u_{1},-u_{2},-u_{3}\right)=-f_{1}\left(t, u_{1}, u_{2}, u_{3}\right)
$$

Under the assumptions $\left(\mathrm{A}_{1}^{\prime}\right) \sim\left(\mathrm{A}_{2}^{\prime}\right)$, similar to Theorem 3.1, it is easy to see that the corresponding energy functional of the system (3.25) is

$$
I(x)=\int_{0}^{2 \gamma \tau}\left[\frac{1}{2}\left(\left|x^{\prime}(t)\right|^{2}+|x(t)|^{2}\right)-\lambda(t) \int_{0}^{x(t-\tau)} g_{1}(t, x(t), \omega) d \omega\right] d t .
$$

THeOrem 3.8. Under the assumptions $\left(\mathrm{A}_{1}^{\prime}\right) \sim\left(\mathrm{A}_{2}^{\prime}\right)$, and the function $g_{1}\left(t, u_{1}, u_{2}\right)$ satisfying the following conditions:

$\left(\mathrm{F}_{1}\right) \lim _{|u| \rightarrow 0}\left(\int_{0}^{x(t-\tau)} g_{1}(t, x(t), \omega) d \omega /|u|^{2}\right)=1$, where $|u|=\sqrt{u_{1}^{2}+u_{2}^{2}} ;$

$\left(\mathrm{F}_{2}\right)$ there exists an $\alpha>0$ such that $g_{1}\left(t, u_{1}, \alpha\right) \leq 0$, for all $\left(t, u_{1}\right) \in[0, \tau] \times(R \backslash[-\alpha, \alpha])$. Denote $\kappa=\min _{t \in[0, \tau]} \lambda(t)$, then when

$$
\kappa>\frac{n^{2}\left(\pi^{2}+\gamma^{2} \tau^{2}\right)}{4 \gamma \tau^{2}}
$$

the problem (3.25) has at least $2 n$ nontrivial $2 \gamma \tau$-periodic solutions. 
Similar to Theorem 3.1, we may show that Theorem 3.8 holds by mean of $Z_{2}$ group index theory, that is, Lemma 1.5.

Proof. Let

$$
h\left(t, u_{1}, u_{2}\right)= \begin{cases}g_{1}\left(t, u_{1}, \alpha\right), & u_{2}>\alpha, \\ g_{1}\left(t, u_{1}, u_{2}\right), & |u| \leq \alpha, \\ g_{1}\left(t, u_{1},-\alpha\right), & u_{2}<-\alpha,\end{cases}
$$

so $h\left(t,-u_{1},-u_{2}\right)=-h\left(t, u_{1}, u_{2}\right)$ is obvious. Let us consider the functional defined on $H_{2 \gamma \tau}^{1}$,

$$
I(x)=\int_{0}^{2 \gamma \tau}\left[\frac{1}{2}\left(\left|x^{\prime}(t)\right|^{2}+|x(t)|^{2}\right)-\lambda(t) \int_{0}^{x(t-\tau)} h(t, x(t), \omega) d \omega\right] d t
$$

First, we show that $I(x)$ has a lower bound.

By the periodicity $x(t), x(t-\tau)$, one gets $\max _{t \in[0,2 \gamma \tau]}|x(t)|=\max _{t \in[0,2 \gamma \tau]}|x(t-\tau)|$. Then, we have $\max _{t \in[0,2 \gamma \tau]}|x(t)|<\alpha$ when $\max _{t \in[0,2 \gamma \tau]}|x(t-\tau)|<\alpha$. On the other hand, by $\left(\mathrm{F}_{2}\right)$, we get $x(t-\tau) h(t, x(t), x(t-\tau)) \leq 0$ when $|x(t-\tau)| \geq \alpha$. So, $\int_{0}^{2 \gamma \tau}\left(\int_{0}^{\alpha} \mid h(t, x(t)\right.$, $\omega) d \omega \mid) d t$ is bounded. Denote $M=\int_{0}^{2 \gamma \tau}\left(\int_{0}^{\alpha}|h(t, x(t), \omega) d \omega|\right) d t$, and $L=\max _{t \in[0, \tau]} \lambda(t)$, then we get

$$
I(x)=\frac{1}{2}\|x\|^{2}-\int_{0}^{2 \gamma \tau} \lambda(t) \int_{0}^{x(t-\tau)} h(t, x(t), \omega) d \omega \geq \frac{1}{2}\|x\|^{2}-L M .
$$

So, $I(x)$ has a lower bound, by the condition $\left(\mathrm{P}_{1}\right)$ of Lemma 1.5 , we get $i_{2}(I)=0$.

Secondly, we will show that $I(x)$ satisfies the P. S. condition. Let $\left\{x_{n}\right\} \subset H_{2 \gamma \tau}^{1}$, and the constants $c_{1}, c_{2}$ satisfy

$$
\begin{gathered}
c_{1} \leq I\left(x_{n}\right) \leq c_{2}, \\
I^{\prime}\left(x_{n}\right) \longrightarrow 0, \quad(n \longrightarrow \infty) .
\end{gathered}
$$

By (3.32), we know

$$
\|x\|_{H_{2 \gamma \tau}^{1}} \leq \sqrt{2 L M+2 c_{2}} \text {. }
$$

So, $\left\|x_{n}\right\|_{H_{2 \gamma \tau}^{1}}$ is bounded. Similarly to the proof of Lemma 3.2, it is easy to see $I(x)$ satisfies the P. S. condition.

Finally, we show that Theorem 3.8 holds by Lemma 1.5.

Denote $\beta_{k}(t)=(\gamma \tau / k \pi) \cos (k \pi / \gamma \tau) t, k=1,2,3, \ldots, n$, then

$$
\begin{aligned}
& \int_{0}^{2 \gamma \tau}\left|\beta_{k}(t)\right|^{2} d t=\frac{\gamma^{2} \tau^{2}}{k^{2} \pi^{2}} \gamma \tau, \\
& \int_{0}^{2 \gamma \tau}\left|\beta_{k}^{\prime}(t)\right|^{2} d t=\gamma \tau .
\end{aligned}
$$


Define the $n$-dimensional space

$$
E_{n}=\operatorname{span}\left\{\beta_{1}(t), \beta_{2}(t), \ldots, \beta_{n}(t)\right\}
$$

It is obvious that $E_{n}$ is a symmetric set. Suppose $\rho>0$, then

$$
E_{n} \bigcap S_{\rho}=\left\{\sum_{k=0}^{n} b_{k} \beta_{k} \mid \sum_{k=0}^{n} b_{k}^{2} \gamma \tau\left(1+\frac{\gamma^{2} \tau^{2}}{k^{2} \pi^{2}}\right)=\rho^{2}\right\} .
$$

On the other hand, we may choose $\varepsilon$ such that $0<\varepsilon<\kappa n^{2} \pi^{2} / \gamma^{2} \tau^{2}\left(2 \gamma^{2} \tau^{2} / n^{2}-\left(\pi^{2}+\right.\right.$ $\left.\left.\gamma^{2} \tau^{2}\right) / \kappa\right)$. By $\left(\mathrm{F}_{2}\right)$, we know that there exists $\delta>0$, when $\left\|x_{n}(t)\right\|_{C}^{2}+\|x(t-\tau)\|_{C}^{2} \leq \delta$ (where $\left\|x_{n}(t)\right\|_{C}^{2}=\max _{0 \leq t \leq 2 \gamma \tau}|x(t)|$ ) such that

$$
\begin{aligned}
\lambda(t) \int_{0}^{x(t-\tau)} & h(t, x(t), \omega) d \omega \\
\geq & (\lambda(t)-\varepsilon)\left[|x(t)|^{2}+|x(t-\tau)|^{2}\right] \\
\geq & (\kappa-\varepsilon)\left[|x(t)|^{2}+|x(t-\tau)|^{2}\right], \quad \forall t \in[0,2 \gamma \tau] .
\end{aligned}
$$

So, choose $\rho=\delta$, when $x \in E_{n} \cap S_{\rho}$, we have

$$
\begin{aligned}
I(x) & =\int_{0}^{2 \gamma \tau}\left[\frac{1}{2}\left(\left|x^{\prime}(t)\right|^{2}+|x(t)|^{2}\right)-\lambda(t) \int_{0}^{x(t-\tau)} h(t, x(t), \omega) d \omega\right] d t \\
& \leq \frac{1}{2} \sum_{k=0}^{n} \gamma \tau b_{k}^{2}\left(1+\frac{\gamma^{2} \tau^{2}}{k^{2} \pi^{2}}\right)-(\kappa-\varepsilon) \int_{0}^{2 \gamma \tau}\left[|x(t)|^{2}+|x(t-\tau)|^{2}\right] d t \\
& \leq \frac{1}{2} \sum_{k=0}^{n} \gamma \tau b_{k}^{2}\left(1+\frac{\gamma^{2} \tau^{2}}{k^{2} \pi^{2}}\right)-2(\kappa-\varepsilon) \int_{0}^{2 \gamma \tau}|x(t)|^{2} d t \\
& \leq \frac{1}{2} \sum_{k=0}^{n} \gamma \tau b_{k}^{2}\left(1+\frac{\gamma^{2} \tau^{2}}{k^{2} \pi^{2}}\right)-2(\kappa-\varepsilon) \sum_{k=0}^{n} \gamma \tau b_{k}^{2} \frac{\gamma^{2} \tau^{2}}{k^{2} \pi^{2}} \\
& \leq \frac{1}{2} \sum_{k=0}^{n} \gamma \tau b_{k}^{2}\left(1+\frac{\gamma^{2} \tau^{2}}{\pi^{2}}\right)-2(\kappa-\varepsilon) \sum_{k=0}^{n} \gamma \tau b_{k}^{2} \frac{\gamma^{2} \tau^{2}}{n^{2} \pi^{2}} \\
& \leq \frac{\kappa \gamma \tau}{2 \pi^{2}}\left(\frac{\pi^{2}+\gamma^{2} \tau^{2}}{\kappa}-\frac{4 \gamma^{2} \tau^{2}}{n^{2}}+\varepsilon \frac{\gamma^{2} \tau^{2}}{\kappa n^{2} \pi^{2}}\right)<0 .
\end{aligned}
$$

(The above equality makes use of $\kappa>n^{2}\left(\pi^{2}+\gamma^{2} \tau^{2}\right) / 4 \gamma \tau^{2}$ and $0<\varepsilon<\kappa n^{2} \pi^{2} / \gamma^{2} \tau^{2}\left(2 \gamma^{2} \tau^{2} / n^{2}-\right.$ $\left.\left(\pi^{2}+\gamma^{2} \tau^{2}\right) / \kappa\right)$.)

So, $i_{1}(I) \geq n$. On the other hand, by $\left(\mathrm{A}_{2}^{\prime}\right)$, we know $I(\theta)=0$. By Lemma 1.5 , we have that the problem (3.25) has at least $2 n$ nontrivial $2 \gamma \tau$-periodic solutions. 
2700 Periodic solutions of mixed-type FDE

Example 3.9. We consider periodic solutions of the following mixed-type differential equations:

$$
x^{\prime \prime}(t-\tau)+4\left[1+\sin ^{2} \frac{\pi t}{\tau}\right]\left(x^{2}(t)+2 x^{2}(t-\tau)+x^{2}(t-2 \tau)\right) x(t-\tau)-x(t-\tau)=0 .
$$

By

$$
\begin{aligned}
f(t, x(t), x(t-\tau), x(t-2 \tau)) \\
=4\left[1+\sin ^{2} \frac{\pi t}{\tau}\right]\left(x^{2}(t)+2 x^{2}(t-\tau)+x^{2}(t-2 \tau)\right) x(t-\tau)-x(t-\tau) \\
=4\left[1+\sin ^{2} \frac{\pi t}{\tau}\right]\left(x^{2}(t)+x^{2}(t-\tau)\right) x(t-\tau) \\
\quad+4\left[1+\sin ^{2} \frac{\pi t}{\tau}\right]\left(x^{2}(t-\tau)+x^{2}(t-2 \tau)\right) x(t-\tau)-x(t-\tau),
\end{aligned}
$$

we have

$$
g(t, x(t), x(t-\tau))=4\left[1+\sin ^{2} \frac{\pi t}{\tau}\right]\left(x^{2}(t)+x^{2}(t-\tau)\right) x(t-\tau)
$$

that is,

$$
g\left(t, u_{1}, u_{2}\right)=4\left[1+\sin ^{2} \frac{\pi t}{\tau}\right]\left(u_{1}^{2}+u_{2}^{2}\right) u_{2}
$$

So, we get

$$
\begin{aligned}
\int_{0}^{x(t-\tau)} g(t, x(t), \omega) d \omega & =\int_{0}^{x(t-\tau)} 4\left[1+\sin ^{2} \frac{\pi t}{\tau}\right]\left(x^{2}(t)+\omega\right) \omega d \omega \\
& =\left[1+\sin ^{2} \frac{\pi t}{\tau}\right]\left(x^{2}(t)+x^{2}(t-\tau)\right)^{2}
\end{aligned}
$$

It is easy to verify $g\left(t, u_{1}, u_{2}\right)$ satisfies $\left(\mathrm{A}_{1}\right) \sim\left(\mathrm{A}_{2}\right)$ and $\left(\mathrm{C}_{1}\right) \sim\left(\mathrm{C}_{2}\right)$. So, by Theorem 3.1 , we know the mixed-type differential (3.40) has an infinite number nontrivial $2 \gamma \tau$-periodic solution.

Example 3.10. Let us consider the system

$$
x^{\prime \prime}(t-\tau)+\lambda(t) f_{1}(t, x(t), x(t-\tau))=x(t-\tau)
$$

where

$$
\begin{aligned}
& f_{1}(t, x(t), x(t-\tau), x(t-2 \tau)) \\
& \quad=4 x(t-\tau)-4\left[1+\sin ^{2} \frac{\pi t}{\tau}\right]\left(x^{2}(t)+2 x^{2}(t-\tau)+x^{2}(t-2 \tau)\right) x(t-\tau),
\end{aligned}
$$


then we get

$$
g_{1}\left(t, u_{1}, u_{2}\right)=2 u_{2}-4\left[1+\sin ^{2} \frac{\pi t}{\tau}\right]\left(u_{1}^{2}+u_{2}^{2}\right) u_{2}
$$

So, we have

$$
\begin{aligned}
& \int_{0}^{x(t-\tau)}\left(2 u_{2}-4\left[1+\sin ^{2} \frac{\pi t}{\tau}\right]\left(x(t)^{2}+u_{2}^{2}\right) u_{2}\right) d u_{2} \\
& =x^{2}(t)+x^{2}(t-\tau)-\left[1+\sin ^{2} \frac{\pi t}{\tau}\right]\left(x^{2}(t)+x^{2}(t-\tau)\right)^{2} \\
& \lim _{|u| \rightarrow 0} \frac{\int_{0}^{u_{2}} g_{1}\left(t, u_{1}, \omega\right) d \omega}{|u|^{2}}=\lim _{|u| \rightarrow 0} \frac{u_{1}^{2}+u_{2}^{2}-\left[1+\sin ^{2}(\pi t / \tau)\right]\left(u_{1}^{2}+u_{2}^{2}\right)^{2}}{u_{1}^{2}+u_{2}^{2}}=1 .
\end{aligned}
$$

By Theorem 3.8, when (3.29) the problem (3.45) has at least $2 n$ nontrivial $2 \gamma \tau$-periodic solutions.

\section{Acknowledgments}

The authors would like to thank the referee for useful remarks on the preliminary version of this paper. The project is supported by NNSF of China (10471155), the Foundation of the Guangdong province, Natural Science Committee (031608), and a specific Foundation for Ph.D. Specialities of Educational Department of China (20020558092).

\section{References}

[1] K. C. Chang, Critical Point Theory and Its Applications, Xiandai Shuxue Congshu, Shanghai Kexue Jishu Chubanshe, Shanghai, 1986.

[2] D. Guo, J. Sun, and Z. Liu, Means of Functions in Nonlinear Ordinary Differential Equations, Shandong Kexue Jishu Chubanshe, Shandong, 1995.

[3] Z.-C. Han, Periodic solutions of a class of dynamical systems of second order, J. Differential Equations 90 (1991), no. 2, 408-417.

[4] Z. Q. Han, 2 $\pi$-periodic solutions to ordinary differential systems at resonance, Acta Math. Sinica 43 (2000), no. 4, 639-644 (Chinese).

[5] S. P. Lu and W. G. Ge, Periodic solutions of second order differential equations with deviating arguments, Acta Math. Sinica 45 (2002), no. 4, 811-818 (Chinese).

[6] J. Mawhin and M. Willem, Critical Point Theory and Hamiltonian Systems, Applied Mathematical Sciences, vol. 74, Springer, New York, 1989.

[7] P. H. Rabinowitz, Minimax Methods in Critical Point Theory with Applications to Differential Equations, CBMS Regional Conference Series in Mathematics, vol. 65, American Mathematical Society, Rhode Island, 1986.

[8] G. Q. Wang, Periodic solutions to second-order neutral equations, Appl. Math. J. Chinese Univ. Ser. A 8 (1993), no. 3, 251-254 (Chinese).

[9] G. Q. Wang and J. R. Yan, Existence of periodic solutions for second order nonlinear neutral delay equations, Acta Math. Sinica 47 (2004), no. 2, 379-384 (Chinese).

[10] Y.-T. Xu and Z.-M. Guo, Applications of a $Z_{p}$ index theory to periodic solutions for a class of functional differential equations, J. Math. Anal. Appl. 257 (2001), no. 1, 189-205.

[11] Applications of a geometrical index theory to functional differential equations, Acta Math. Sinica 44 (2001), no. 6, 1027-1036 (Chinese). 


\section{Periodic solutions of mixed-type FDE}

[12] Y. Zhang and Y. Zhang, Periodic solutions to second-order linear neutral differential equations with constant coefficients, Acta Math. Sinica 33 (1990), no. 4, 517-520 (Chinese).

Xiao-Bao Shu: Department of Mathematics, Hunan University, Changsha 410082, China E-mail address: sxb0221@163.com

Yuan-Tong Xu: Department of Mathematics, Zhongshan University, Guangzhou 510275, China E-mail address: xyt@zsu.edu.cn 


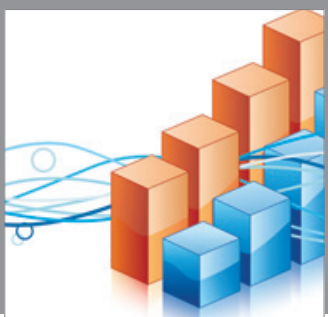

Advances in

Operations Research

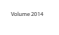

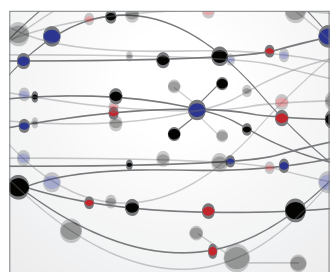

\section{The Scientific} World Journal
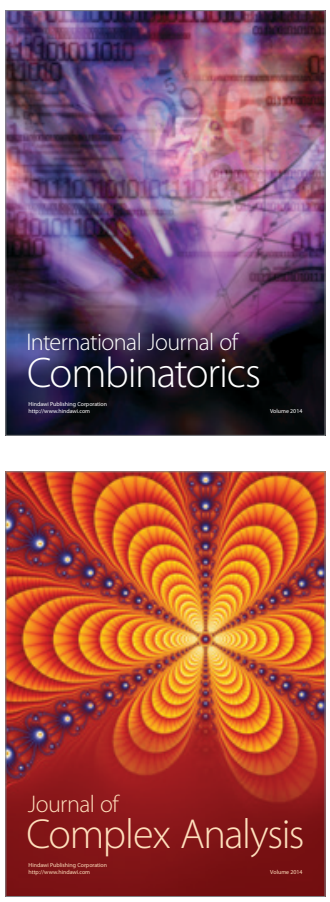

International Journal of

Mathematics and

Mathematical

Sciences
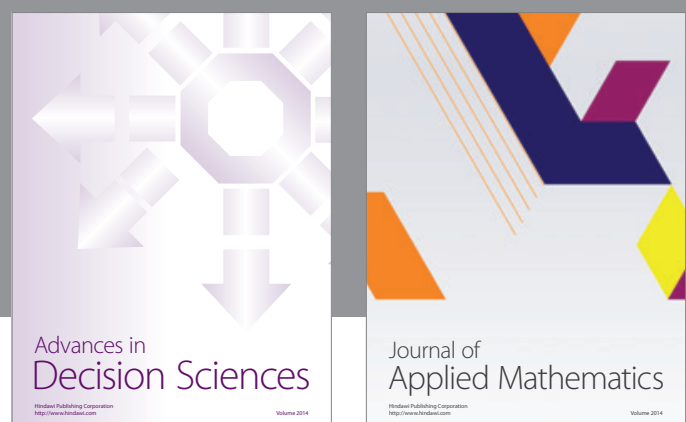

Journal of

Applied Mathematics
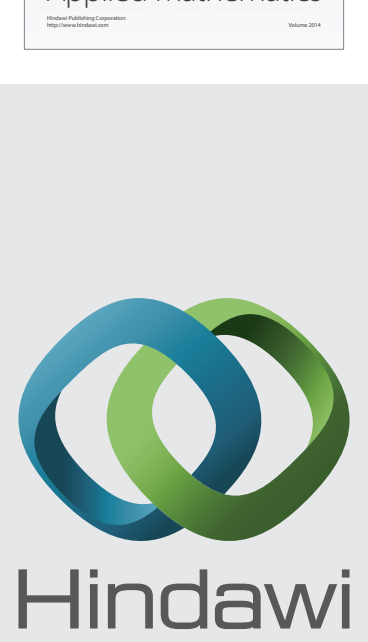

Submit your manuscripts at http://www.hindawi.com
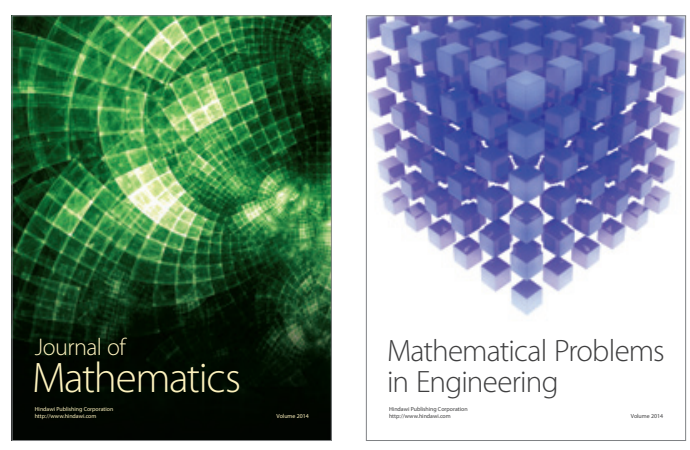

Mathematical Problems in Engineering
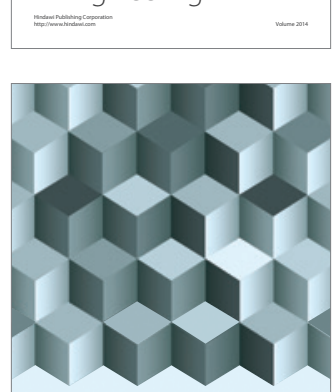

Journal of

Function Spaces
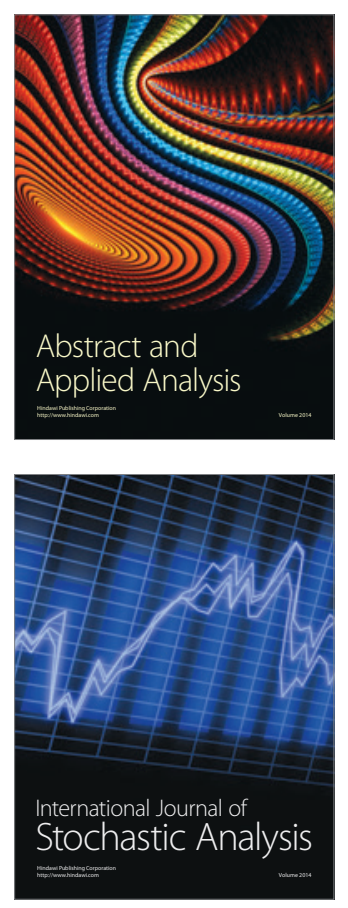

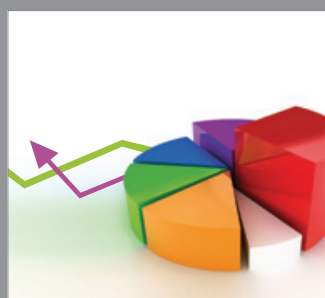

ournal of

Probability and Statistics

Promensencen
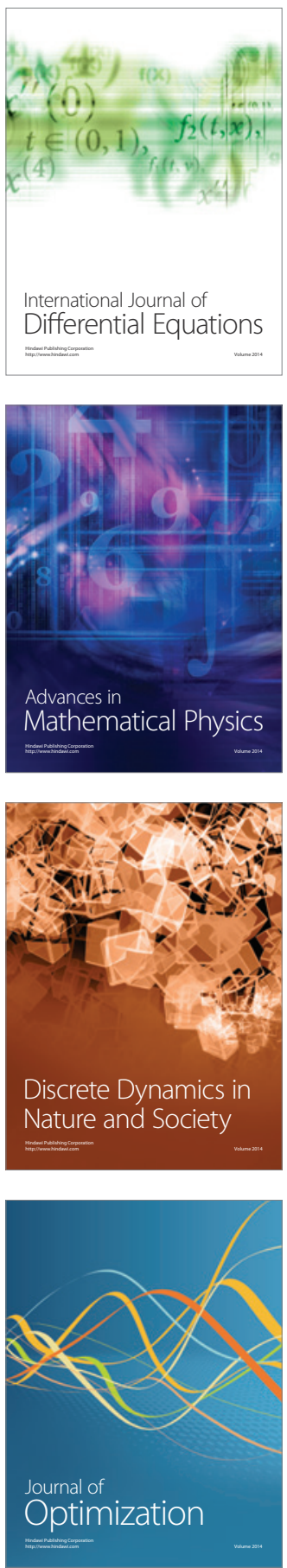